\title{
Revealing the role of VEGFA in clear cell sarcoma of the kidney by protein-protein interaction network and significant pathway analysis
}

\author{
ZHIKUI WANG, ZHAOXIA WANG, ZHONGQI ZHOU and YUEQIN REN
}

\author{
Department of Nephrology, Linyi People's Hospital, Linyi, Shandong 276003, P.R. China
}

Received February 2, 2015; Accepted October 23, 2015

DOI: $10.3892 / \mathrm{ol} .2015 .4006$

\begin{abstract}
Despite clear cell sarcoma of the kidney (CCSK) being the second most common renal tumor in children, its mechanism has not yet been fully investigated. The aim of the present study was to investigate the potential role of vascular endothelial growth factor A (VEGFA) in CCSK development. Following preprocessing of the original GSE2712 data, the differentially-expressed genes (DEGs) between 14 CCSK and 3 fetal kidney samples were identified through Significance Analysis of Microarrays, using the R package. Pathway enrichment analysis was then performed on the DEGs. A protein-protein interaction (PPI) network was constructed using the Search Tool for the Retrieval of Interacting Genes/Proteins database and the DEGs that were enriched in the most significant pathways. Following this, gene ontology analysis was performed on the VEGFA-associated genes, whilst transcription factor binding site analysis was conducted on the hot genes. A total of 2,681 DEGs, including 543 upregulated and 2,138 downregulated genes, were identified, and these were significantly enriched in pathways associated with cancer and focal adhesion. Furthermore, VEGFA, integrin $\beta 1$, integrin $\alpha \mathrm{V}$, v-akt murine thymoma viral oncogene homolog 1 and endothelial growth factor receptor were identified as hot genes in the PPI network. In addition, the upregulated VEGFA-associated genes, cyclin D1 and cyclin-dependent kinase inhibitor $1 \mathrm{~B}$, affected kinase regulation, and the downregulated VEGFA-associated genes, receptor tyrosine-protein kinase erbB-2, mesenchymal-epithelial transition tyrosine kinase receptor and kinase insert domain receptor, were enriched in the protein tyrosine kinase process. It was identified that VEGFA was regulated by restorer of fertility, erythromycin resistance methylase, GA binding protein
\end{abstract}

Correspondence to: Mr. Zhaoxia Wang, Department of Nephrology, Linyi People's Hospital, 27 Jiefang Road, Linyi, Shandong 276003, P.R. China

E-mail: zhaoxiawangzwk@163.com

Key words: clear cell sarcoma of the kidney, differentiallyexpressed genes, pathway analysis, protein-protein interaction network, transcription factor binding site analysis subunit $\alpha$, norepinephrine transporter, nuclear factor $\kappa \mathrm{B}$ and Sp2 transcription factor genes. Overall, VEGFA and its associated genes serve important roles during CCSK development, and alongside transcription factors, they may function as novel therapeutic targets for disease treatment.

\section{Introduction}

Clear cell sarcoma of the kidney (CCSK) is a rare, malignant childhood tumor occurring most frequently in the second and third years of life (1). CCSK has a wide histological spectrum and may often resemble other pediatric renal tumors, resulting in considerable diagnostic challenges. The clinical presentation of CCSK includes a large tumor size (mean diameter, $11.3 \mathrm{~cm}$ ), a mucoid texture, foci of necrosis and prominent cyst formation (2). Typically, the therapeutic drugs used to treat CCSK include vincristine, doxorubicin and dactinomycin (3). The diagnosis of CCSK is important for its prevention, management and treatment. However, only $68 \%$ of CCSKs were correctly identified by institutional pathologists during the last cooperative group clinical protocol (4). Therefore, there is an urgent requirement to identify novel biomarkers for CCSK diagnosis.

Over the previous decades, genetic studies regarding CCSK have been limited and the underlying mechanism of the disease consequently remains unclear. Dhamne et al (5) reported that cyclin D1 (CCND1) may be a central molecule in the pathogenesis of CCSK, primarily regulated by nuclear factor $\kappa \mathrm{B}(\mathrm{NF}-\kappa \mathrm{B})$ cells. Forkhead box D1 and cbp/p300-interacting transactivator, with Glu/Asp-rich carboxy-terminal domain 1, are highly expressed in CCSK, with oxidative-stress-responsive kinase 1, an early embryonic marker, also expressed at high levels in CCSK and at greater levels than observed in normal kidneys or Wilms' tumors (WT) (6). Little et al (7) identified the dysregulation of epidermal growth factor receptor (EGFR) at multiple levels within CCSK. Boo et al (8) detected the expression of vascular endothelial growth factor (VEGF) and angiopoietin 2 in CCSK tumor cells, indicating that angiogenesis in CCSK may involve these proteins. Furthermore, Short et al (9) reported that CCSK is typically hypermethylated at the $\mathrm{CpG}$ of thrombospondin-1, which is a suspected angiogenic factor, suggesting that angiogenesis serves a role in CCSK. Therefore, the specific role of angiogenesis in CCSK development 
requires full investigation, with the aim of novel targets being identified.

In the present study, the mechanism of CCSK was investigated using pathway analysis and protein-protein interaction (PPI) networks, whilst the role of VEGFA in CCSK development was predicted through gene ontology (GO) and transcription factor binding site (TFBS) analyses. The results demonstrated that VEGFA and its associated genes serve important roles during CCSK development and progression.

\section{Materials and methods}

Affymetrix chip data. The Gene Expression Omnibus (GEO) database located in the National Center for Biotechnology Information is currently the largest fully public gene expression resource, including 214,268 samples and 4,500 platforms (10). The chip dataset GSE2712, which includes 14 CCSK, 15 WT and 3 fetal kidney (FK) samples, was downloaded from GEO (4). In the present study, the gene expression patterns of 14 CCSKs were compared to 3 FK samples through the use of oligonucleotide arrays. The platform used was the GPL96 (HG-U133A) GeneChip ${ }^{\circledR}$ Human Genome 2.0 U133A Array (Affymetrix, Inc., Santa Clara, CA, USA).

Data preprocessing and the identification of differentially-expressed genes (DEGs). The Affy $\mathrm{R}$ package is a package of functions and classes for the analysis of oligonucleotide arrays (11). Data preprocessing was performed using robust multi-array average in the Affy $\mathrm{R}$ package, including background-adjusted, normalized and log-transformed values (12). The original CEL file data was then transformed to probe-level. Following this, the probe-level data was converted to gene symbols by the perl procedure (13). When several probes corresponded to one gene symbol, the gene expression value was calculated as the mean of each probe. DEGs between the 14 CCSKs and 3 FK samples were identified by Significance Analysis of Microarrays using the R package, with llog fold-changel $>1.5$ and false discovery rate $<0.05(14,15)$.

Functional analysis. In the present study, the Database for Annotation, Visualization and Integrated Discovery (DAVID) was applied to conduct Kyoto Encyclopedia of Genes and Genomes (KEGG) analyses of the DEGs. The DAVID bioinformatics resource consists of an integrated biological knowledge base and analytical tools aimed at systematically extracting biological meaning from large gene or protein lists (16). KEGG is a knowledge base that allows for the systematic analysis of gene functions (17). For the present study, the significant KEGG pathways, in which the DEGs were enriched, were screened out with $\mathrm{P}<0.05$ and the most notable pathways were selected for further analysis.

PPI analysis. PPI analysis was conducted on the DEGs that were enriched in the most significant KEGG pathways through the use of the Search Tool for the Retrieval of Interacting Genes/Proteins (STRING). The genes with PPI $\geq 30$ were described as hot genes. The STRING database (http://string-db.org/) provides global protein interactions and associations (18). The PPI network was then visualized using Cytoscape software (19).
GO analysis. The up- and downregulated genes that interacted with hot genes were run through GO analysis by DAVID. GO terms are significantly overrepresented in a set of genes from three aspects, consisting of the cellular component, molecular function (MF) and biological process (BP) (20). The significant GO terms with $\mathrm{P}<0.05$ were selected for further analysis.

TFBS analysis. TFBS analysis implements objects representing specific profile matrices, binding sites and sets thereof, pattern generators and pattern database interfaces (21). In the present study, the whole genome rVista (http://genome.lbl. gov/cgi-bin/WGRVistaInput5.pl?cfg_dir=gp_r4099_169) was used to select TFBS for hot genes (22). TFBS analysis was performed from 5,000 bp to the transcription start site and the significant TFBS was selected with $\mathrm{P}<0.01$. Finally, the result was visualized by Cytoscape software.

\section{Results}

Identified DEGs and significant pathways. Based on the aforementioned analysis, a total of 2,681 DEGs between 14 CCSK and $3 \mathrm{FK}$ samples were identified, including 543 upregulated and 2,138 downregulated genes. Furthermore, a total of 33 overexpressed genes, including wingless-type MMTV integration site family, member 5A and fibroblast growth factor receptor 1 , were demonstrated to be significantly enriched in the cancer-associated pathways $\left(\mathrm{P}=1.35 \times 10^{-6}\right)$. Additionally, a total of 50 downregulated genes, including VEGFA and p21-activated kinase 4 , were significantly enriched in focal adhesion $\left(\mathrm{P}=1.03 \times 10^{-5}\right)$ (Table I).

PPI network. Combined with the STRING database, PPI analysis was performed on the 33 upregulated and 50 downregulated genes enriched in the two pathways. The PPI network is presented in Fig. 1, with five hot genes screened out, including three overexpressed genes [VEGFA, integrin $\alpha \mathrm{V}$ (ITG $\alpha \mathrm{V}$ ) and integrin $\beta 1$ (ITG $\beta 1$ )] and two downregulated genes [v-akt murine thymoma viral oncogene homolog 1 (AKT1) and EGFR].

GO analysis for VEGFA-associated genes. As presented in Table II, the VEGFA-associated upregulated genes [cyclin D1 and cyclin-dependent kinase inhibitor 1B (CDKN1B)] were significantly enriched in three MF terms, including cyclin-dependent protein kinase regulator activity $(\mathrm{P}=0.0137)$, protein kinase regulator activity $(\mathrm{P}=0.0408)$ and kinase regulator activity $(\mathrm{P}=0.0408)$. Furthermore, downregulated genes (receptor tyrosine-protein kinase erbB-2 (ERBB2), mesenchymal-epithelial transition (MET) tyrosine kinase receptor and kinase insert domain receptor (KDR)] markedly disturbed two MF terms and three BP terms. The most significant $\mathrm{MF}$ and $\mathrm{BP}$ terms were transmembrane receptor protein tyrosine kinase activity $\left(\mathrm{P}=3.39 \times 10^{-4}\right)$ and transmembrane receptor protein tyrosine kinase signaling pathway $\left(\mathrm{P}=7.93 \times 10^{-4}\right)$.

Results of TFBS analysis. TFBS analysis was performed on the four hot genes of VEGFA, ITG $\beta 1$, EGFR and AKTI. The results are presented in Fig. 2. VEGFA was demonstrated to 
Table I. Most significant pathways in which the differentially-expressed genes were enriched $(\mathrm{P}<0.05)$.

\begin{tabular}{|c|c|c|c|c|}
\hline Term & Count & P-value & Genes & Gene regulation \\
\hline hsa05200: Pathways in cancer & 33 & $1.35 \times 10^{-6}$ & $\begin{array}{l}\text { WNT5A, FGFR1, FGF18, WNT5B, } \\
\text { FGF9, PDGFA, FOXO1, FGF13, } \\
\text { ZBTB16, KIT, GLI2, GLI1, AKT1, } \\
\text { IGF1R, WNT4, CASP3, CASP8, } \\
\text { RUNX1, CEBPA, EGFR, AR, BMP2, } \\
\text { TCF7, CTBP2, EPAS1, CDK6, } \\
\text { CDKN1A, CCND1, CDKN1B, } \\
\text { PDGFRA, PTCH1, WNT11, PIAS1 }\end{array}$ & Upregulated \\
\hline hsa04510: Focal adhesion & 50 & $1.03 \times 10^{-5}$ & $\begin{array}{l}\text { PAK4, ILK, PDGFC, AKT3, ACTN4, } \\
\text { ROCK1, ROCK2, FLNB, VASP, } \\
\text { PRKCB, PPP1CA, LAMC3, VEGFA } \\
\text { MAPK3, RELN, LAMC1, COL1A1, } \\
\text { PARVB, PARVA, IBSP, CAV2, ERBB2, } \\
\text { TNC, ITGB1, PXN, MYL9, LAMB2, } \\
\text { DOCK1, ITGAV, SOS2, COL6A3, } \\
\text { THBS1, SPP1, COL4A4, VAV3, MET, } \\
\text { ITGA2, MYLPF, MYL12B, ITGA3, } \\
\text { MYL12A, ITGA4, CAPN2, COL4A6, } \\
\text { KDR, LAMA1, LAMA5, ITGA8, } \\
\text { ITGA7, MYLK }\end{array}$ & Downregulated \\
\hline
\end{tabular}

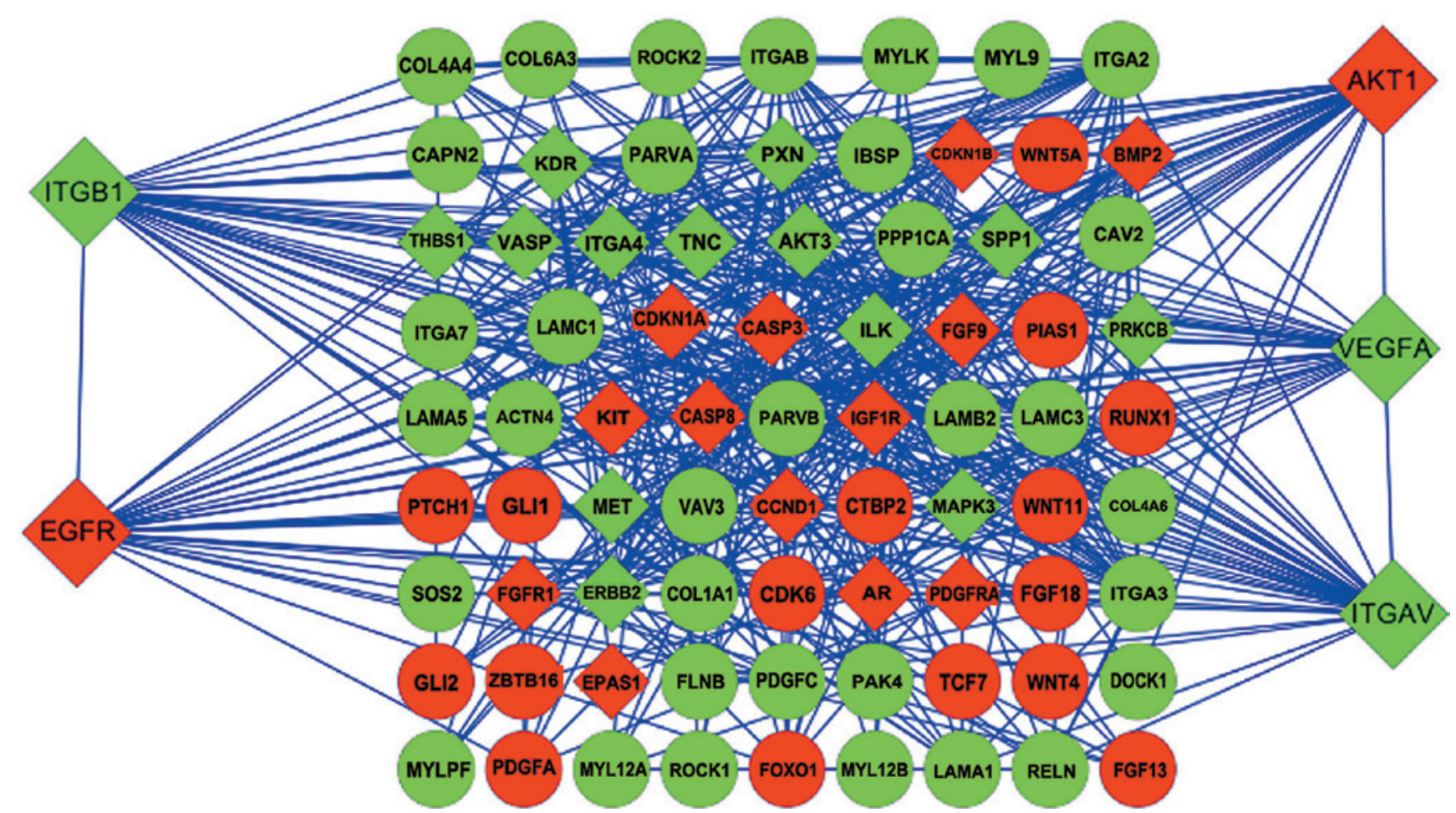

Figure 1. PPI network of the differentially-expressed genes enriched in the two significant pathways. Diamonds indicate genes associated with vascular endothelial growth factor A (VEGFA); circles indicate genes not associated with VEGFA; red indicates upregulated genes; green indicates downregulated genes; and larger diamonds indicate hot genes with $>30$ related genes.

be regulated by six TFs, including ETS domain-containing transcription factor (ERF), erythromycin resistance methylase
(ERM), GA binding protein subunit $\alpha(\mathrm{GABP} \alpha)$, norepinephrine transporter $(\mathrm{Net}), \mathrm{NF}-\kappa \mathrm{B}$ and $\mathrm{Sp} 2$ transcription factor. 
Table II. Significant GO terms for vascular endothelial growth factor A-related genes $(\mathrm{P}<0.05)$.

\begin{tabular}{lllll}
\hline Category & \multicolumn{1}{c}{ Term } & P-value & \multicolumn{1}{c}{ Genes } & Regulation \\
\hline GOTERM_MF_FAT & $\begin{array}{l}\text { GO:0016538 cyclin-dependent protein } \\
\text { kinase regulator activity }\end{array}$ & $1.37 \times 10^{-2}$ & CCND1, CDKN1B & Upregulated \\
GOTERM_MF_FAT & $\begin{array}{l}\text { GO:0019887 protein kinase regulator } \\
\text { activity }\end{array}$ & $4.08 \times 10^{-2}$ & CCND1, CDKN1B & Upregulated \\
GOTERM_MF_FAT & $\begin{array}{l}\text { GO:0019207 kinase regulator activity } \\
\text { GO:0004714 transmembrane receptor } \\
\text { pOTERM_MF_FAT }\end{array}$ & $\begin{array}{l}4.08 \times 10^{-2} \\
\text { protein tyrosine kinase activity }\end{array}$ & $\begin{array}{c}\text { CCND1, CDKN1B } \\
\text { ERBB2, MET, KDR }\end{array}$ & $\begin{array}{c}\text { Upregulated } \\
\text { Downregulated }\end{array}$ \\
GOTERM_MF_FAT & $\begin{array}{l}\text { GO:0004713 protein tyrosine kinase } \\
\text { activity }\end{array}$ & $1.48 \times 10^{-3}$ & ERBB2, MET, KDR & Downregulated \\
GOTERM_BP_FAT & $\begin{array}{l}\text { GO:0007169 transmembrane receptor } \\
\text { protein tyrosine kinase signaling pathway }\end{array}$ & $7.93 \times 10^{-4}$ & ERBB2, MET, KDR & Downregulated \\
GOTERM_BP_FAT & $\begin{array}{l}\text { GO:0007167 enzyme-linked receptor } \\
\text { protein signaling pathway }\end{array}$ & $1.71 \times 10^{-3}$ & ERBB2, MET, KDR & Downregulated \\
GOTERM_BP_FAT & GO:0006928 cell motion & $5.01 \times 10^{-3}$ & ERBB2, VASP, KDR & Downregulated
\end{tabular}

MF, molecular function; BP, biological process; GO, gene ontology.

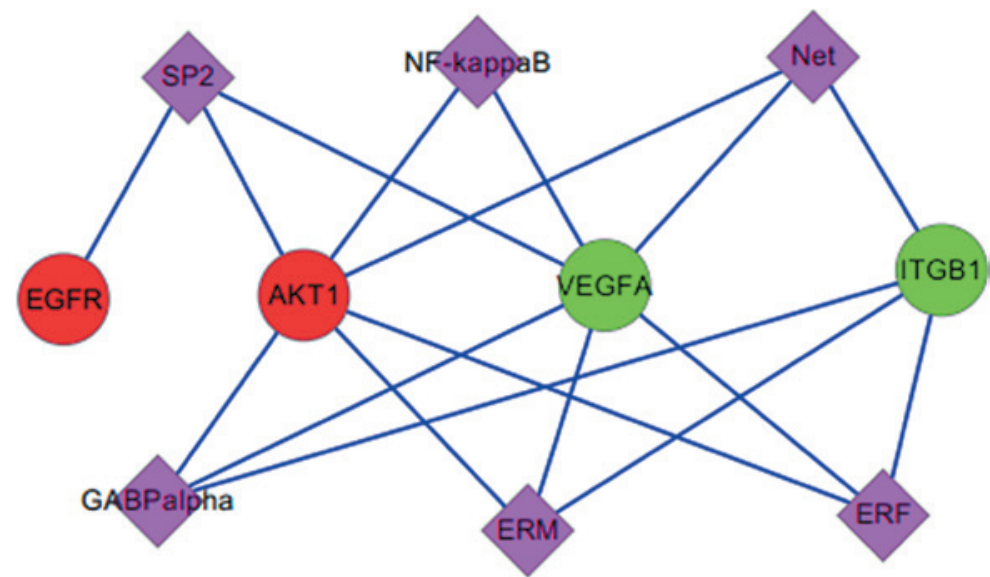

Figure 2. Transcription factor binding site analysis for hot genes. Diamonds indicate overexpressed TF names; red circles indicate upregulated genes; green circles indicate downregulated genes and blue lines indicate interactions between transcription factors and hot genes.

Similarly, the upregulated gene, AKT1, was also regulated by the same six TFs.

\section{Discussion}

CCSK was originally described in 1970, and often develops in children $<3$ years (23). CCSK has presented significant diagnostic and clinical challenges, thus numerous studies have aimed to investigate the mechanism of CCSK and also to identify markers for the diagnosis and treatment of the disease. In the present study, the underlying pathogenesis of CCSK was predicted through the use of bioinformatic approaches, with novel markers singled out for use in therapeutics.

A total of 2,681 DEGs, between 14 CCSK and 3 FK samples, were identified. In these DEGs, 2,138 genes were identified to be downregulated in CCSK samples, including VEGFA, whilst 543 were overexpressed. Such results suggest that these dysregulations may induce the development of CCSK, particularly in the downregulated genes. Furthermore, it was demonstrated that 33 upregulated genes were significantly enriched in cancer-associated pathways $\left(\mathrm{P}=1.35 \times 10^{-6}\right)$, whilst 50 downregulated genes, including VEGFA, were enriched in focal adhesion pathways $\left(\mathrm{P}=1.03 \times 10^{-5}\right)$. These findings indicate that the dysregulation of these genes may induce CCSK by disturbing these two pathways. Previous studies have demonstrated that these pathways serve important roles in cancer development and progression $(24,25)$, which is consistent with the results from the current study.

In addition, a PPI network was constructed and hot genes were identified. By using DEGs enriched in the two most significant pathways and the STRING database, the PPI network was established and five hot genes were singled out. In the network, numerous genes interacted with VEGFA, which included the two upregulated hot genes AKT1 and EGFR, and the two downregulated genes ITG $\beta 1$ and ITG $\alpha$ V. VEGFA is a member of the platelet-derived growth factor/VEGF family and encodes a protein that is typically identified as a disulfide-linked homodimer; it is also an angiogenic factor that is 
required for tumor growth and metastasis (26). The protein it encodes is a glycosylated mitogen, specifically acting on endothelial cells, with its effects including increased vascular permeability, the promotion of cell migration, the induction of angiogenesis, vasculogenesis and endothelial cell growth, and the inhibition of apoptosis. EGFR, a growth factor receptor tyrosine kinase, has been detected as a common component of multiple types of cancer (27). AKT1 has been widely studied and is demonstrated to be frequently overexpressed in human cancer, with the ability to accelerate cell proliferation and suppress programmed cell death (28). Hunt et al (29) reported that the aberrant activation and expression of ITG $\beta 1$ has been identified in a number of malignancies. IT $\alpha \mathrm{V}$ is associated with numerous types of cancer, including colon, gastric and breast cancer (30). Based on previous studies, these five hot genes have been widely investigated and detected in multiple tumors. Notably, in the present study, the dysregulation of these genes was also identified in CCSK, suggesting that the five hot genes serve important roles in CCSK development.

Furthermore, the functions of VEGFA-associated genes were analyzed by GO. The two upregulated genes, CCND1 and CDKN1B, were involved in kinase regulation, whilst the downregulated genes, ERBB2, MET and KDR, were significantly enriched in protein tyrosine kinase regulation. CCND1 is involved in several human malignancies, including breast, colorectal and non-small cell lung cancer (31). Variant genotypes of CDKN1B, encoding p27 KIP1, are associated with an increased risk of breast cancer, prostate carcinoma and human hepatocellular carcinoma (32). Overexpression of ERBB2, a receptor-like tyrosine kinase, has been detected in several types of human carcinomas (33). The MET tyrosine kinase receptor promotes tissue remodeling, which subsequently induces organ homeostasis, cancer metastasis and wound repair (34). Jang et al (35) demonstrated that VEGF and KDR serve important roles in angiogenesis, contributing to the development and progression of numerous tumors. The results of the present study demonstrated that these genes were associated with CCSK development, which is consistent with previous studies.

Through TFBS analysis, six TFs that regulated VEGFA, including ERF, ERM, GABP $\alpha$, Net, NF- $\kappa \mathrm{B}$ and SP2, were identified. These six TFs have the ability to regulate VEGFA expression, indicating that the conditions of these TFs may induce the upregulation, downregulation or normal expression of VEGFA, with the downregulated VEGFA believed to subsequently induce CCSK. Boo et al (8) reported the expression of VEGF in CCSK tumor cells, indicating that angiogenesis in CCSK may involve VEGF. However, the associations between VEGF and TFs in CCSK have not yet been investigated. Therefore, to the best of our knowledge, the present study predicts that these TFs are important in CCSK development, diagnosis and treatment for the first time.

In conclusion, the current study investigated the underlying mechanism of CCSK and identified novel diagnostic markers for the disease. Downregulated VEGFA and its interacting genes may initiate CCSK development by interrupting two different pathways, kinase regulation and protein tyrosine kinase metabolism. Additionally, VEGFA, ITGB1, ATK1 and EGFR would be the diagnostic markers for CCSK, while ERF, ERM, GABP $\alpha$, Net, NF- $\kappa$ B and SP2 may function as targets for CCSK therapy. The present study focused on an original insight regarding the pathogenesis of CCSK and identified novel markers, as well as targets for the disease. However, due to the disadvantages and limitations of bioinformatic approaches, further investigation is required to verify such results.

\section{References}

1. Hartman RJ Jr, Welchons DR, Teot L, Chow J and Cendron M: Incidental capture of rarely diagnosed pediatric tumor: An infant boy with clear cell sarcoma of the kidney. Urology 82: 1416-1418, 2013.

2. Argani P, Perlman EJ, Breslow NE, Browning NG, Green DM, D'Angio GJ and Beckwith JB: Clear cell sarcoma of the kidney: A review of 351 cases from the National Wilms Tumor Study Group Pathology Center. Am J Surg Pathol 24: 4-18, 2000.

3. Seibel NL, Li S, Breslow NE, Beckwith JB, Green DM, Haase GM, Ritchey ML, Thomas PR, Grundy PE, Finklestein JZ, et al: Effect of duration of treatment on treatment outcome for patients with clear-cell sarcoma of the kidney: A report from the National Wilms' Tumor Study Group. J Clin Oncol 22: 468-473, 2004.

4. Cutcliffe C, Kersey D, Huang CC, Zeng Y, Walterhouse D and Perlman EJ; Renal Tumor Committee of the Children's Oncology Group: Clear cell sarcoma of the kidney: Up-regulation of neural markers with activation of the sonic hedgehog and Akt pathways. Clin Cancer Res 11: 7986-7994, 2005.

5. Dhamne S, Brown RE, Covinsky M, Dhamne C, Eldin K and Tatevian N: Clear cell sarcoma of kidney: Morphoproteomic analysis reveals genomic correlates and therapeutic options. Pediatr Dev Pathol 16: 20-27, 2013.

6. Karlsson J, Holmquist Mengelbier L, Ciornei CD, Naranjo A, O'Sullivan MJ and Gisselsson D: Clear cell sarcoma of the kidney demonstrates an embryonic signature indicative of a primitive nephrogenic origin. Genes Chromosomes Cancer 53: 381-391, 2014.

7. Little SE, Bax DA, Rodriguez-Pinilla M, Natrajan R, Messahel B, Pritchard-Jones K, Vujanic GM, Reis-Filho JS and Jones C: Multifaceted dysregulation of the epidermal growth factor receptor pathway in clear cell sarcoma of the kidney. Clin Cancer Res 13: 4360-4364, 2007.

8. Boo YJ, Fisher JC, Haley MJ, Cowles RA, Kandel JJ and Yamashiro DJ: Vascular characterization of clear cell sarcoma of the kidney in a child: A case report and review. J Pediatr Surg 44: 2031-2036, 2009.

9. Short SS, Zmora O, Hunter CJ, Wang L, Siegel S and Ford HR: Large clear cell sarcoma of the kidney mistaken as Wilms' tumor. J Pediatr Surg Rep 1: 235-238, 2013.

10. Barrett T, Wilhite SE, Ledoux P, Evangelista C, Kim IF, Tomashevsky M, Marshall KA, Phillippy KH, Sherman PM, Holko M, et al: NCBI GEO: Archive for functional genomics data sets - update. Nucleic Acids Res 41: D991-D995, 2013.

11. Gautier L, Cope L, Bolstad BM, and Irizarry RA: affy - analysis of Affymetrix GeneChip data at the probe level. Bioinformatics 20: 307-315, 2004.

12. Irizarry RA, Hobbs B, Collin F, Beazer-Barclay YD, Antonellis KJ, Scherf $U$ and Speed TP: Exploration, normalization, and summaries of high density oligonucleotide array probe level data. Biostatistics 4: 249-264, 2003

13. Tiengo A, Barbarini N, Troiani S, Rusconi L and Magni P: A Perl procedure for protein identification by peptide mass fingerprinting. BMC Bioinformatics 10 (Suppl 12): S11, 2009.

14. Efron B, Tibshirani R, Storey JD and Tusher V: Empirical Bayes analysis of a microarray experiment. J Am Stat Assoc 96: 1151-1160, 2001.

15. Efron B and Tibshirani R: Empirical bayes methods and false discovery rates for microarrays. Genet Epidemiol 23: 70-86, 2002.

16. Huang DW, Sherman BT, Tan Q, Collins JR, Alvord WG, Roayaei J, Stephens R, Baseler MW, Lane HC and Lempicki RA: The DAVID Gene Functional Classification Tool: A novel biological module-centric algorithm to functionally analyze large gene lists. Genome Biol 8: R183, 2007.

17. Kanehisa M and Goto S: KEGG: Kyoto encyclopedia of genes and genomes. Nucleic Acids Res 28: 27-30, 2000.

18. Franceschini A, Szklarczyk D, Frankild S, Kuhn M, Simonovic M, Roth A, Lin J, Minguez P, Bork P, von Mering C and Jensen LJ: STRING v9. 1: Protein-protein interaction networks, with increased coverage and integration. Nucleic Acids Res 41: D808-D815, 2013. 
19. Saito R, Smoot ME, Ono K, Ruscheinski J, Wang P-L, Lotia S, Pico AR, Bader GD and Ideker T: A travel guide to Cytoscape plugins. Nat Methods 9: 1069-1076, 2012.

20. Ashburner M, Ball CA, Blake JA, Botstein D, Butler H, Cherry JM, Davis AP, Dolinski K, Dwight SS, Eppig JT, et al Gene Ontology: Tool for the unification of biology. The Gene Ontology Consortium. Nat Genet 25: 25-29, 2000.

21. Lenhard B and Wasserman WW: TFBS: Computational framework for transcription factor binding site analysis. Bioinformatics 18: 1135-1136, 2002.

22. Loots GG, Ovcharenko I, Pachter L, Dubchak I and Rubin EM rVista for comparative sequence-based discovery of functional transcription factor binding sites. Genome Res 12: 832-839, 2002.

23. Kidd JM: Exclusion of certain renal neoplasms from the category of Wilms tumor. Am J Pathol 59: 16a, 1970.

24. McLean GW, Carragher NO, Avizienyte E, Evans J, Brunton VG and Frame MC: The role of focal-adhesion kinase in cancer - a new therapeutic opportunity. Nat Rev Cancer 5: 505-515, 2005.

25. Rodriguez-Gonzalez A, Lin T, Ikeda AK, Simms-Waldrip T, $\mathrm{Fu} \mathrm{C}$ and Sakamoto KM: Role of the aggresome pathway in cancer: Targeting histone deacetylase 6-dependent protein degradation. Cancer Res 68: 2557-2560, 2008.

26. Hanrahan V, Currie MJ, Gunningham SP, Morrin HR, Scott PA, Robinson BA and Fox SB: The angiogenic switch for vascular endothelial growth factor (VEGF)-A, VEGF-B, VEGF-C, and VEGF-D in the adenoma-carcinoma sequence during colorectal cancer progression. J Pathol 200: 183-194, 2003.

27. Nicholson RI, Gee JM, and Harper ME: EGFR and cancer prognosis. Eur J Cancer 37 (Suppl 4): S9-S15, 2001.
28. Liu H, Radisky DC, Nelson CM, Zhang H, Fata JE, Roth RA and Bissell MJ: Mechanism of Akt1 inhibition of breast cancer cell invasion reveals a protumorigenic role for TSC2. Proc Natl Acad Sci USA 103: 4134-4139, 2006

29. Hunt S, Jones AV, Hinsley EE, Whawell SA and Lambert DW: MicroRNA-124 suppresses oral squamous cell carcinoma motility by targeting ITGB1. FEBS Lett 585: 187-192, 2011.

30. Daemen A, Gevaert O, De Bie T, Debucquoy A, Machiels J-P, De Moor B and Haustermans K. Integrating microarray and proteomics data to predict the response on cetuximab in patients with rectal cancer. Pac Symp Biocomput 13: 166-177, 2008.

31. Porter TR, Richards FM, Houlston RS, Evans DG, Jankowski JA Macdonald F, Norbury G, Payne SJ, Fisher SA, Tomlinson I and Maher ER: Contribution of cyclin d1 (CCND1) and E-cadherin (CDH1) polymorphisms to familial and sporadic colorectal cancer. Oncogene 21: 1928-1933, 2002.

32. Fornari F, Gramantieri L, Ferracin M, Veronese A, Sabbioni S, Calin GA, Grazi GL, Giovannini C, Croce CM, Bolondi L and Negrini M: MiR-221 controls CDKN1C/p57 and CDKN1B/p27 expression in human hepatocellular carcinoma. Oncogene 27: 5651-5661, 2008.

33. Harari D and Yarden Y: Molecular mechanisms underlying ErbB2/ HER2 action in breast cancer. Oncogene 19: 6102-6114, 2000.

34. Trusolino L, Bertotti A and Comoglio PM: MET signalling: Principles and functions in development, organ regeneration and cancer. Nat Rev Mol Cell Biol 11: 834-848, 2010.

35. Jang MJ, Jeon YJ, Kim JW, Cho YK, Lee SK, Hwang SG, Oh D and Kim NK: Association of VEGF and KDR single nucleotide polymorphisms with colorectal cancer susceptibility in Koreans. Mol Carcinog 52 (Suppl 1): E60-E69, 2013. 